\section{P205 Implementation and Outcome of a Farmers' Market Targeting WIC Farmers' Market Nutrition Program (FMNP) Participants: A Case Study Using CBPR Approach}

Lanae Ball, MS, lanae.ball@gmail.com, University of North Carolina at Greensboro, 109 Gresham Avenue, Durham, NC 27704; J. Dharod, PhD; K. Johnston, Catawba County Public Health; A. McCauley,; T. Paul, BSPH; J. Byrd, RD

Objective: The main objectives of this study are: 1) to describe the overall process and key strategies involved in implementing a farmers' market at a WIC office; and, 2) to assess improvement in redemption rate of FMNP coupons among WIC participants by improving farmers' market access.

Study Design, Setting, Participants, and Intervention: A case study design using different formative methods documented the process and key inputs in farmers' market implementation. Catawba County Public Health (CCPH) WIC clinic served as the farmers' market site. Local farmers, program administrators, and community advocates were reached in documenting the imple- mentation process. FMNP participants were the target audience. All study protocols were approved by the UNCG IRB.

Outcome, Measures and Analysis: Multiple measurement methods included direct observation and semi structured interviews with staff and farmers $(n=13)$. Grounded theory informed interview analysis. The primary outcome measure was FMNP coupon redemption rate. Rates from 2007-2013 were averaged and compared using a weighted ranking system (\% redemption * \% eligible individuals).

Results: A community partnership among WIC, Eat Smart Move More, local farmers, UNCG, and CCPH was key in implementing an onsite farmers' market. The market occurred Thursdays from May-October 2013, and farmers offered a variety of local produce. The average redemption rate from 2007-2013 was 51.4\% (rank of 10). The 2013 redemption rate increased from $51.3 \%$ to $62.9 \%$ (rank of 3 ).

Conclusions and Implications: This study highlights the importance of leveraging resources at the community level to address barriers to FMNP redemption. Locating farmers' markets at WIC clinics, in particular, can reduce barriers for FMNP participants and increase redemption.

Funding: Eat Smart Move More 